\title{
Osmoregulation in a Proline-producing Strain of Serratia marcescens
}

\author{
By MASAKI SUGIURA* AND MASAHIKO KISUMI \\ Research Laboratory of Applied Biochemistry, Tanabe Seiyaku Co. Ltd, 3-16-89, Kashima, \\ Yodogawa-ku, Osaka 532, Japan
}

(Received 6 February 1985; revised 29 May 1985)

\begin{abstract}
A proline-producing strain of Serratia marcescens grew more rapidly than the wild-type strain in a medium of high osmolarity due to high concentrations of $\mathrm{NaCl}, \mathrm{KCl}, \mathrm{Na}_{2} \mathrm{SO}_{4},\left(\mathrm{NH}_{4}\right)_{2} \mathrm{HPO}_{4}$, sodium glutamate, glucose or sucrose. Growth inhibition by $\mathrm{NaCl}$ was partially reversed by proline in the wild-type strain, and by glutamate and proline in the proline-producing strain. Intracellular proline and glutamate concentrations under conditions of high osmolarity were studied. The wild-type strain accumulated endogenously synthesized glutamate, and concentrated proline taken up from the external medium. In contrast, the proline-producing strain accumulated a large amount of endogenously synthesized proline. This increased proline content contributes to the osmotolerance of the proline-producing strain. The growth inhibition by $\mathrm{NaCl}$ was also reversed by glycinebetaine in $S$. marcescens wild-type and proline-producing strains.
\end{abstract}

\section{INTRODUCTION}

The osmotic strength of the medium is one of the important parameters influencing the growth of micro-organisms. Most micro-organisms, except for halophiles, show optimal growth at low osmotic strength and the internal osmolarity is controlled by the accumulation of amino acids (Tempest et al., 1970), inorganic cations (Thompson \& MacLeod, 1974), polyhydroxy alcohols (Brown, 1974) or carbohydrates (Roller \& Anagnostopoulos, 1982). With amino acids, proline or glutamate functions as the most effective osmobalancer when non-halophilic bacteria are subjected to conditions of high osmolarity. Measures (1975) reported that Gram-negative species mainly accumulate glutamate in response to osmotic stress, and that those organisms which accumulate proline are Gram-positive. Csonka (1981) obtained osmotolerant mutants of Salmonella typhimurium which overproduced proline owing to regulatory mutations affecting proline biosynthesis.

Three enzymes are involved in the synthesis of proline from glutamate in bacteria (Umbarger, 1978): glutamate kinase, glutamate- $\gamma$-semialdehyde dehydrogenase and pyrroline-5-carboxylate reductase. The major regulatory point of proline biosynthesis is the feedback control of glutamate kinase (Adams \& Frank, 1980). We have obtained proline-producing strains among proline analogue-resistant mutants of Serratia marcescens (Sugiura et al., 1985). A 3,4dehydroproline-resistant mutant, SP105, derived from a proline oxidase-deficient mutant, produced about $5 \mathrm{mg}$ L-proline per $\mathrm{ml}$ of medium. A thiazolidine-2-carboxylate-resistant mutant, SP126, derived from strain SP105, produced $20 \mathrm{mg}$ L-proline per $\mathrm{ml}$. Using the prolineproducing strain, we have examined the role of proline in the osmoregulatory mechanisms of $S$. marcescens.

\section{METHODS}

Bacterial strains and media. The strains used were $S$. marcescens Sr4l strain 8000 (wild strain; Matsumoto et al., 1975); strain SP103, a proline oxidase-deficient mutant: strain SP105, a 3,4-dehydroproline-resistant mutant derived from strain SP103; and strain SP126, a thiazolidine-4-carboxylate-resistant mutant derived from strain

Abbreviation: GDH, glutamate dehydrogenase.

$0001-2488 \circlearrowright 1985$ SGM 
Table 1. Growth rates of $S$. marcescens wild strain 8000 and proline-producing strain SP126 under conditions of high osmolarity

\begin{tabular}{|c|c|c|}
\hline \multirow[b]{2}{*}{$\begin{array}{l}\text { Additions to } \\
\text { minimal medium (mM) }\end{array}$} & \multicolumn{2}{|c|}{ Specific growth rate* $\left(\mathrm{h}^{-1}\right)$} \\
\hline & SP126 & $\overbrace{8000}$ \\
\hline None & 0.50 & $0 \cdot 68$ \\
\hline $\mathrm{NaCl}(600)$ & $0 \cdot 11$ & $0 \cdot 08$ \\
\hline $\mathrm{KCl}(600)$ & $0 \cdot 11$ & $0 \cdot 08$ \\
\hline $\mathrm{Na}_{2} \mathrm{SO}_{+}(400)$ & $0 \cdot 24$ & $0 \cdot 11$ \\
\hline$\left(\mathrm{NH}_{4}\right)_{2} \mathrm{HPO}_{4}(400)$ & $0 \cdot 20$ & $0 \cdot 11$ \\
\hline Sodium glutamate $(600)$ & 0.17 & 0.05 \\
\hline Glucose $(600)$ & $0 \cdot 43$ & 0.37 \\
\hline Sucrose $(600)$ & 0.50 & 0.43 \\
\hline
\end{tabular}

* Defined as $\ln 2 /$ (mass doubling time, h).

SP105 (Sugiura et al., 1985). Miminal medium (M1 medium) was that of Davis \& Mingioli (1950), modified by omitting the citrate and increasing the glucose concentration to $0.5 \%$ as decribed previously (Sugiura \& $\mathrm{K}$ isumi, 1984). Nutrient medium contained $0.5 \%$ glucose, $1.0 \%$ polypeptone, $0.3 \%$ meat extract, $1.0 \%$ yeast extract and $0.5 \% \mathrm{NaCl}, \mathrm{pH} 7.0$. For the solid medium, $1.5 \%$ agar was added. All amino acids used were L-configuration.

Growth experiments. These were done with a Hitachi automated recording incubator system (Chibata et al., 1977). Cells were grown at $30^{\circ} \mathrm{C}$ with shaking (140 r.p.m.) in a test tube containing $3 \mathrm{ml} \mathrm{medium}$. Growth was measured as the $\mathrm{OD}_{660}$ every hour. Bacteria grew exponentially until an $\mathrm{OD}_{660}$ of 0.6 was reached. The generation time was expressed as the mass doubling time, calculated during exponential growth at optical density values between $0 \cdot 1$ and $0 \cdot 5$. The specific growth rate was defined as $\ln 2 /($ mass doubling time, $h$ ).

Analytical procedures. The influence of growth conditions on the intracellular contents of proline and glutamate was assessed as follows: $150 \mathrm{ml} \mathrm{Ml}$ medium in a $500 \mathrm{ml}$ flask was inoculated with a loopful of cells grown on a nutrient slant, and incubated at $30^{\circ} \mathrm{C}$ with reciprocal shaking $\left(140\right.$ oscillations $\mathrm{min}^{-1}, 7 \mathrm{~cm}$ stroke $)$. Growth was estimated by measuring the $\mathrm{OD}_{600}$ with a Hitachi photoelectric photometer (EPO-B type). To adjust the osmolarity of the medium to $0.3 \mathrm{M}-\mathrm{NaCl}, 15 \mathrm{ml} 3.3 \mathrm{M}-\mathrm{NaCl}$ was added to the $\mathrm{Ml}$ medium during exponential growth and cells were further cultured for 0.5 to $1.0 \mathrm{~h}$ after this addition. In certain experiments, proline or glutamate were also added, either separately or together. At the end of the exponential growth phase $[0.5$ to $0.7 \mathrm{mg}$ dry wt cells ( $\mathrm{ml} \mathrm{culture})^{-1}$ ], cells were harvested by centrifugation $(5000 \mathrm{~g}, 5 \mathrm{~min})$ and washed rapidly once with $100 \mathrm{ml}$ cold fresh medium. When $\mathrm{NaCl}$ medium was used, cells were also washed with cold $\mathrm{Ml}$ medium containing $0.3 \mathrm{M}-\mathrm{NaCl}$. Thereafter, cells were suspended in $3 \mathrm{ml}$ water. A portion of the suspension was diluted with water and the $\mathrm{OD}_{600}$ measured; the extent of growth was then calculated from a standard curve; an optical density of 0.10 corresponded to $160 \mu \mathrm{g}$ dry wt cells $\mathrm{ml}^{-1}$, as described previously (Sugiura et al., 1981). The suspension was boiled for $10 \mathrm{~min}$ and centrifuged at $20000 \mathrm{~g}$ for $3 \mathrm{~min}$. The proline and glutamate contents of the supernatant were then determined by microbioassay with Leuconostoc mesenteroides P-60 (Steele et al., 1949). The contents were expressed as millimolar intracelluiar concentrations using a value of cell water based on the assumption that the wet weight of cells is four times the dry weight (Tempest et al., 1970).

Enzyme assay. Exponentially growing cells in M1 medium were harvested by centrifugation, disrupted with a sonic oscillator and centrifuged as described previously (Sugiura \& Kisumi, 1985). The cell-free supernatants were then assayed for protein by the method of Lowry, and for glutamate dehydrogenase (GDH) by measuring the reduction of NADPH by the method of Dendinger et al. (1980). Specific activity was expressed as $\mu$ mol product $\min ^{-1}(\mathrm{mg} \text { protein })^{-1}$.

\section{RESULTS}

Growth of a proline-producing strain in media of high osmolarity

Growth of two strains of $S$. marcescens, wild strain 8000 and a proline-producing strain SP126, was determined in media with increased osmolarity due to both electrolytes and non-electrolytes (Table 1). In minimal medium, strain SP126 grew more slowly than strain 8000 , probably because of the abnormal metabolic flow due to the release of feedback controls in proline biosynthesis. In all media of high osmolarity, however, strain SP126 grew more rapidly, i.e. 


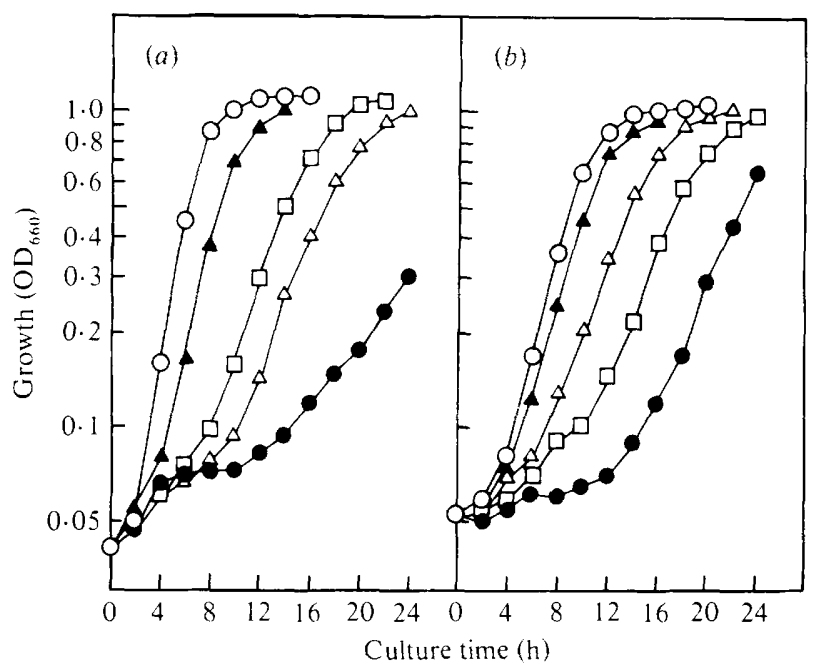

Fig. 1. Growth inhibition by $\mathrm{NaC}$ and its reversal by proline, glutamate and glycinebetaine in wild and proline-producing strains of $S$. marcescens. (a) Wild strain $8000:(b)$ proline-producing strain SP126. The strains were grown in minimal medium with the following additions: $\bigcirc$, none:, $600 \mathrm{~mm}-\mathrm{NaCl}$; $\square, 600 \mathrm{mu}-\mathrm{NaCl}+1 \mathrm{~mm}$-proline: $\triangle, 600 \mathrm{~mm}-\mathrm{NaCl}+1 \mathrm{~mm}$-glutamate; $\boldsymbol{\Delta} .600 \mathrm{~mm}-\mathrm{NaCl}+1 \mathrm{~mm}-$ glycinebetaine.

strain SP126 was more tolerant than strain 8000 to the osmotic stress imposed by $\mathrm{NaCl}, \mathrm{KCl}$, sodium glutamate, glucose, sucrose, $\mathrm{Na}_{2} \mathrm{SO}_{4}$ or $\left(\mathrm{NH}_{4}\right)_{2} \mathrm{HPO}_{4}$. These results suggest the presence of an osmoregulatory mechanism induced by proline in $S$. marcescens.

\section{Reversal of growth inhibition induced by $\mathrm{NaCl}$}

Csonka (1981) reported that proline could reverse the osmotic inhibition in Salmonella typhimurium; we confirmed this phenomenon in Serratia marcescens. Of 16 common amino acids tested (alanine, arginine, aspartate, cysteine, glutamate, glycine, histidine, isoleucine, leucine, lysine, methionine, phenylalanine, proline, serine, threonine and valine; $1 \mathrm{~mm}$ each), only proline and glutamate reversed the growth inhibition (Fig. 1). In wild strain $8000,600 \mathrm{~mm}-\mathrm{NaCl}$ strongly inhibited growth. Addition of $1 \mathrm{~mm}$-proline or glutamate alleviated the growth inhibition, although the reversal by either amino acid was incomplete. Increasing the concentration of either amino acid to $10 \mathrm{~mm}$ did not enhance the reversal (data not shown). In the proline-producing strain SP126, reversal of growth inhibition by glutamate was greater than that by proline. Again increasing the concentration of either amino acid did not enhance the reversal. These results suggest that proline and glutamate function as osmoregulators in $S$. marcescens.

Apart from amino acids, it was recently reported that methylated quaternary ammonium compounds, such as glycinebetaine, could protect some enteric bacteria against osmotic stress (LeRudulier \& Valentine, 1982). The growth inhibition of $S$. marcescens in media of high osmolarity was also relieved by exogenous glycinebetaine, and the reversal by glycinebetaine was found in both proline-producing and non-producing strains (Fig. 1). These results indicate that glycinebetaine is also an osmoregulator in $S$. marcescens.

Changes in intracellular glutamate and proline concentrations under conditions of high osmolarity

Intracellular glutamate and proline concentrations in $S$. marcescens were determined (Table 2). In minimal medium, the intracellular glutamate concentration of strain 8000 was about $8 \mathrm{~mm}$, which was about 10 -fold greater than the proline concentration. Neither glutamate nor 
Table 2. Changes in intracellular glutamate and proline concentrations in two $S$. marcescens strains under conditions of high osmolarity

\begin{tabular}{|c|c|c|c|c|}
\hline \multirow[b]{3}{*}{$\begin{array}{l}\text { Additions to minimal } \\
\text { medium (mM) }\end{array}$} & \multicolumn{4}{|c|}{ Intracellular concentrations ( $\mathrm{mM}$ ) } \\
\hline & \multicolumn{2}{|c|}{8000} & \multicolumn{2}{|c|}{ SPI26 } \\
\hline & $\overbrace{\text { Glu }}$ & Pro & Glu & Pro \\
\hline None & $7 \cdot 8$ & $0 \cdot 8$ & $4 \cdot 8$ & 18 \\
\hline Glu (10) & $7 \cdot 2$ & 0.7 & $5 \cdot 2$ & 22 \\
\hline Pro $(10)$ & $6 \cdot 8$ & $1 \cdot 0$ & $4 \cdot 5$ & 24 \\
\hline $\mathrm{NaCl}(300)$ & 79 & 0.8 & 40 & 97 \\
\hline $\mathrm{NaCl}(300)+$ Glu $(10)$ & 103 & $1 \cdot 0$ & 38 & 110 \\
\hline $\mathrm{NaCl}(300)+$ Pro $(1)$ & 72 & 91 & 35 & 120 \\
\hline $\mathrm{NaCl}(300)+$ Pro $(10)$ & 75 & 88 & 34 & 110 \\
\hline
\end{tabular}

Table 3. Changes in glutamate dehydrogenase activity in $S$. marcescens strain 8000 under various conditions

\begin{tabular}{|c|c|c|c|}
\hline $\begin{array}{l}\text { Addition to } \\
\text { minimal } \\
\text { medium (mM) }\end{array}$ & $\begin{array}{l}\text { Addition to } \\
\text { reaction } \\
\text { mixture (mM) }\end{array}$ & $\begin{array}{l}\mathrm{pH} \text { of } \\
\text { reaction } \\
\text { mixture* }\end{array}$ & $\begin{array}{c}\text { Relative } \\
\text { activity } \\
(\%)\end{array}$ \\
\hline None & None & $\begin{array}{l}7 \cdot 25 \\
7 \cdot 5 \\
7 \cdot 75 \\
8 \cdot 0 \\
8 \cdot 25 \\
8 \cdot 5 \\
8 \cdot 75\end{array}$ & $\begin{array}{l}61 \\
73 \\
76 \\
100(0.095) \dagger \\
101 \\
100 \\
90\end{array}$ \\
\hline None & $\begin{array}{l}\mathrm{NaCl}(100) \\
\mathrm{NaCl}(300) \\
\mathrm{NaCl}(500)\end{array}$ & $\begin{array}{l}8 \cdot 0 \\
8 \cdot 0 \\
8 \cdot 0\end{array}$ & $\begin{array}{l}142 \\
145 \\
130\end{array}$ \\
\hline $\mathrm{NaCl}(300)$ & None & $8 \cdot 0$ & 145 \\
\hline
\end{tabular}

proline concentrations were increased by addition of the amino acids to the medium. When the osmolarity of the medium was increased by addition of $300 \mathrm{mM}-\mathrm{NaCl}$, the intracellular glutamate concentration increased significantly to about $80 \mathrm{~mm}$; the proline concentration, however, was not changed. The addition of glutamate to the high osmolarity medium slightly increased the intracellular glutamate concentration, but had no effect on the proline concentration. On the other hand, the addition of $1 \mathrm{mM}$ - or $10 \mathrm{~mm}$-proline in the presence of $300 \mathrm{~mm}-\mathrm{NaCl}$ increased the proline concentration 100 -fold. These results suggest that proline transport into the cells is stimulated under conditions of high osmolarity, and that growth inhibition by $\mathrm{NaCl}$ is alleviated by an increase in the intracellular proline concentration. The further addition of glutamate had no effect on either glutamate or proline concentration. Addition of other amino acids, such as arginine, threonine or isoleucine, did not influence the intracellular concentrations of amino acids, either in minimal medium or after addition of $\mathrm{NaCl}$ (data not shown). The intracellular proline and glutamate concentrations in strain SP103, a proline oxidase-deficient mutant, were similar to those in strain 8000 (data not shown).

In order to identify the roles of proline and glutamate in osmoregulation by $S$. marcescens, intracellular concentrations of both amino acids in the proline-producing strain SP126 under conditions of high osmolarity were determined (Table 2). In minimal medium, the intracellular proline concentration was $18 \mathrm{~mm}$, which is some 20 -fold greater than that of the wild-type strain. In contrast, the glutamate concentration was slightly lower, at $5 \mathrm{~mm}$. Both concentrations were little influenced by addition of glutamate or proline to the minimal medium. In a medium of high osmolarity, however, the intracellular proline concentration increased to $100 \mathrm{~mm}$, whereas the glutamate concentration only increased to $40 \mathrm{~mm}$. Neither amino acid intracellular concentration was affected by the further addition of either glutamate or proline. 
The reason for the increase of intracellular glutamate concentration under high osmotic conditions was examined. A hypothesis that GDH, a key enzyme for glutamate biosynthesis in bacteria (Rosenfeld \& Brenchley, 1983), was activated by alkalization of intracellular pH due to addition of $\mathrm{NaCl}$ was proposed for Aerobacter aerogenes (Brown \& Stanley, 1972). We examined the GDH activity of $S$. marcescens wild strain 8000 at various $\mathrm{pH}$ values and under conditions of high osmolarity (Table 3 ). The enzyme had a broad optimum at $\mathrm{pH} 8 \cdot 0$ to $8 \cdot 5$. It was slightly activated by 100 and $300 \mathrm{~mm}-\mathrm{NaCl}$, and its activity in extracts of cells grown in $300 \mathrm{~mm}-\mathrm{NaCl}$ medium was 1.5 times greater than that in extracts of cells grown in minimal medium. This increase in GDH activity and content may contribute to the increase in intracellular glutamate concentration under conditions of high osmolarity.

\section{DISCUSSION}

A proline-producing strain of Serratia marcescens was more osmotolerant than the wild-type strain under conditions of high osmotic stress induced by both electrolytes and non-electrolytes. The intracellular proline concentration of the proline-producing strain was 20 times higher than that of the wild-type strain, suggesting that the enhanced osmotolerance might be a consequence of the high intracellular concentration of proline.

The intracellular glutamate concentration of the wild-type strain of $S$. marcescens increased 10 -fold when cells were grown in a medium of high osmolarity, but the proline concentration did not change under these conditions. While Measures (1970) reported the increases of intracellular glutamate and proline concentrations in $S$. marcescens under conditions of high osmolarity, it was not clear whether both amino acids were synthesized internally or were transported from the medium, because a nutrient broth was used in the experiment. Using a chemically defined medium, Brown \& Stanley (1972) reported an increase of intracellular glutamate concentration in $A$. aerogenes and of proline in Bacillus subtilis. More recently, Csonka (1981) made a more precise examination of the changes in intracellular amino acid concentrations in wild-type and proline-producing strains of Salmonella typhimurium under conditions of high osmolarity: glutamate and glutamine concentrations increased, but proline concentration did not change in the wild-type strain. The reason for the increase of intracellular glutamate concentration in these micro-organisms was unclear, although some hypotheses were proposed. Since the GDH of Serratia marcescens had a broad $\mathrm{pH}$ optimum, the activation of GDH by alkalization of intracellular $\mathrm{pH}$ is improbable. The addition of $300 \mathrm{~mm}-\mathrm{NaCl}$ to the reaction mixture and culture medium increased the GDH activity by about 1.5 times. It is not clear, however, whether these slight increases in GDH activity and/or content contribute to the significant increase of intracellular glutamate concentration.

The intracellular proline concentration of the wild-type strain increased about 100-fold when cells were grown in a proline-containing high osmolarity medium. This result indicates that the wild-type strain of $S$. marcescens accumulates exogenous proline in the cells to oppose the osmotic stress. This is probably the reason why proline reverses the osmotic inhibition more effectively than glutamate in wild-type strains. A third proline permease, functioning under conditions of high osmotic stress, was reported in Salmonella typhimurium (Csonka, 1982), suggesting the possibility of the presence of a similar proline permease in Serratia marcescens. We have found that the sensitivity to proline analogues was significantly increased in a high osmotic stress medium (Sugiura \& Kisumi, 1985). Changes in the intracellular analogue concentration under conditions of high osmolarity are now under investigation.

The intracellular proline concentration of the proline-producing strain increased to about $100 \mathrm{~mm}$ during high osmotic stress, indicating that proline synthesis was activated under these conditions. Since the feedback control of proline biosynthesis is released in proline-producing strains, the increased proline concentration might be due to the rapid conversion of glutamate, the concentration of which increased during high osmotic stress. Therefore proline, as well as glutamate, is important for osmoregulation in the proline-producing strain. These results agree with those in Salmonella typhimurium (Csonka, 1981), in which proline levels in the proline- 
producing strain harbouring the mutant allele pro-74 increased up to 22 -fold in response to $0.65 \mathrm{M}-\mathrm{NaCl}$.

In conclusion, the wild-type strain of Serratia marcescens withstands osmotic stress by increasing the intracellular concentration of endogenously synthesized glutamate in minimal medium, and by increasing the intracellular concentration of proline taken up from the external medium in a proline-containing medium. In contrast, a proline-producing strain of $S$. marcescens responds to the stress by increasing the intracellular proline and glutamate concentrations in either the presence or the absence of proline. In addition to these osmoregulatory mechanisms involving amino acids, another osmoregulatory mechanism involving glycinebetaine is also present in $S$. marcescens.

We wish to thank Ichiro Chibata, Senior Managing Director, Research and Development Headquarters of our company, for encouragement. We also thank Yasuko Nonoguchi for technical assistance.

\section{REFERENCES}

Adams, E. \& Frank, L. (1980). Metabolism of proline and hydroxyproline. Annual Review of Biochemistry 49, 1005-1061.

Brown, A. D. (1974). Microbial water relations: features of the intracellular composition of sugartolerant yeasts. Journal of Bacteriology 118, 769-777.

Brown, C. M. \& Stanley, S. O. (1972). Environmentmediated changes in the cellular content of the 'pool' constituents and their associated changes in cell physiology. Journal of Applied Chemistry and Biotechnology 22, 363-389.

Chibata, I., Itoh, H. \& Morimoto, T. (1977). Automated recording incubator for tube culture of microorganisms. Chemical Economy and Engineering Review 9, 11-15.

Csonka, L. N. (1981). Proline over-production results in enhanced osmotolerance in Salmonella typhimurium. Molecular and General Genetics 182, 82-86.

Csonka, L. N. (1982). A third L-proline permease in Salmonella typhimurium which functions in media of elevated osmotic strength. Journal of Bacteriology 151, 1433-1443

Davis, B. D. \& Mingioli, E. S. (1950). Mutants of Escherichia coli requiring methionine or vitamin $\mathrm{B}_{12}$. Journal of Bacteriology 60, 17-28.

Dendinger, S. H., Patil, L. G. \& Brenchley, J. E. (1980). Salmonella typhimurium mutants with altered glutamate dehydrogenase and glutamate synthase activities. Journal of Bacteriology 141, 190-198.

LeRudulier, D. \& Valentine, R. C. (1982). Genetic engineering in agriculture: osmoregulation. Trends in Biochemical Sciences 7, 431-433.

Matsumoto, H., Hosogaya, S., Suzuki, K . \& Tazaki, T. (1975). Arginine gene cluster of Serratia marcescens. Japanese Journal of Microbiology 19, 35-44.

Measures, J. C. (1975). Role of amino acids in osmoregulation of non-halophilic bacteria. Nature, London 257, 398-400.

Roller, S. D. \& Anagnostopoulos, G. D. (1982). Accumulation of carbohydrate by Escherichia coli
$\mathrm{B} / \mathrm{r} / 1$ during growth at low water activity. Journal of Applied Bacteriology 52, 425-434.

Rosenfeld, S. A. \& BRENChley, J. E. (1983). Regulation of glutamate and glutamine biosynthesis. In Amino Acids: Biosynthesis and Genetic Regulation, pp. 1-17. Edited by K. Herrmann \& R. L. Somerville. Reading, Massachusetts: AddisonWesley.

Steele, B. F., Sauberlich, H. E., Reynolds, M. S. \& Baumann, C. A. (1949). Media for Leuconostoc mesenteroides P-60 and Leuconostoc citrovorum 8081. Journal of Biological Chemistry 177, 533-544.

Sugiura, M. \& Kisumi, M. (1984). Stabilization of a histidine-producing strain of Serratia marcescens. Applied and Environmental Microbiology 48, 43-47.

Sugiura, M. \& Kisumi, M. (1985). Proline-hyperproducing strains of Serratia marcescens: enhancement of proline analog-mediated growth inhibition by increasing osmotic stress. Applied and Environmental Microbiology 49, 782-786.

Sugiura, M., Kisumi, M. \& Chibata, I. (1981). Biosynthetic pathway of $\beta$-methylnorleucine, an antimetabolite produced by Serratia marcescens. Journal of Antibiotics 34, 1283-1289.

Sugiura, M., Takagi, T. \& Kisumi, M. (1985). Proline production by regulatory mutants of Serratia marcescens. Applied Microbiology and Biotechnology 21, 213219.

Tempest, D. W., Meers, J. L. \& Brown, C. M. (1970). Influence of environment on the content and composition of microbial free amino acid pools. Journal of General Microbiology 64, 171-185.

Thompson, J. \& MacLeod, R. A. (1974). Potassium transport and the relationship between intracellular potassium concentration and amino acid uptake by cells of a marine pseudomonad. Journal of Bacteriology 120, 598-603.

UMBARGER, H. E. (1978). Amino acid biosynthesis and its regulation. Annual Review of Biochemistry 49, 1005-1061. 